\title{
NON-ABELIAN GROUPS ACTING ON SEVERI-BRAUER SURFACES
}

\author{
CONSTANTIN SHRAMOV
}

\begin{abstract}
We provide examples of finite non-abelian groups acting on non-trivial Severi-Brauer surfaces.
\end{abstract}

A Severi-Brauer surface over a field $\mathbb{K}$ is a surface that becomes isomorphic to $\mathbb{P}^{2}$ over the algebraic closure of $\mathbb{K}$. Concerning birational automorphisms of non-trivial SeveriBrauer surfaces (i.e. those that are not isomorphic to $\mathbb{P}^{2}$ ) the following is known.

Theorem 1 ([Sh20, Theorem 1.2(ii)]). Let $S$ be a non-trivial Severi-Brauer surface over a field $\mathbb{K}$ of characteristic zero. Then every finite group acting by birational automorphisms of $S$ is either abelian, or contains a normal abelian subgroup of index 3.

The purpose of this note is to prove the following. We denote by $\boldsymbol{\mu}_{n}$ the cyclic group of order $n$.

Theorem 2. Let $p=3 k+1$ be a prime number. Consider the non-trivial homomorphism $\boldsymbol{\mu}_{3} \rightarrow \boldsymbol{\mu}_{3 k} \cong \operatorname{Aut}\left(\boldsymbol{\mu}_{p}\right)$, and let $G_{p}=\boldsymbol{\mu}_{p} \rtimes \boldsymbol{\mu}_{3}$ be the corresponding semi-direct product. Then there exists a number field $\mathbb{K}$ and a non-trivial Severi-Brauer surface $S$ over $\mathbb{K}$ such that the group $\operatorname{Aut}(S)$ contains the group $G_{p}$.

Using the notion of the Jordan constant (see [Pop14, Definition 1]), one can reformulate Theorem 1 by saying that the Jordan constant of the birational automorphism group (and thus also of the usual automorphism group) of a non-trivial Severi-Brauer surface over a field of characteristic zero is at most 3 . In these terms, Theorem 2] states that for a suitable number field $\mathbb{K}$ and a suitable non-trivial Severi-Brauer surface $S$ over $\mathbb{K}$ the Jordan constant of the automorphism group of $S$ (and thus also of its birational automorphism group) attains this bound.

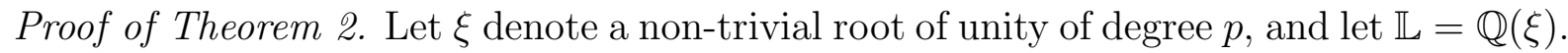
Then $\mathbb{L} / \mathbb{Q}$ is a Galois extension with the Galois group isomorphic to $\boldsymbol{\mu}_{p-1}$, see for instance [Cas67, Lemma III.1.1]. Let $\mathbb{K} \subset \mathbb{L}$ be the field of invariants of the (unique) subgroup $\boldsymbol{\mu}_{3} \subset \operatorname{Gal}(\mathbb{L} / \mathbb{Q})$. Then $\mathbb{L} / \mathbb{K}$ is a Galois extension with the Galois group Gal $(\mathbb{L} / \mathbb{K})$ isomorphic to $\boldsymbol{\mu}_{3}$. A generator $\sigma$ of $\operatorname{Gal}(\mathbb{L} / \mathbb{K})$ sends $\xi \in \mathbb{L}$ to $\xi^{d}$, where $d \neq 1$ is an integer such that $d^{3} \equiv 1(\bmod p)$.

There exists an element $a \in \mathbb{K}$ such that $a$ is not contained in the image of the Galois norm of the field extension $\mathbb{L} / \mathbb{K}$, see [Ste89, Theorem 1(b)]. We consider the cyclic algebra $A$ over $\mathbb{K}$ associated with $\sigma$ and $a$, see [GS06, §2.5] or [GSh18, Exercise 3.1.6]. In other words, $A$ is generated over $\mathbb{K}$ by $\mathbb{L}$ and an element $\alpha$ subject to relations $\alpha^{3}=a$ and

$$
\lambda \alpha=\alpha \sigma(\lambda), \quad \lambda \in \mathbb{L},
$$

so that in particular we have

$$
\xi \alpha=\alpha \xi^{d}
$$


Then $A$ is a central simple algebra. However, $A$ is not a matrix algebra, see for instance [GSh18, Exercise 3.1.6(i)]. Since the dimension of $A$ over $\mathbb{K}$ is 9, we conclude from the theorem of Wedderburn (see [GS06, Theorem 2.1.3]) that $A$ is a division algebra.

The order of $\alpha$ in the multiplicative group $A^{*}$ equals 3 ; since $\alpha \notin \mathbb{K}$, the order of its image $\hat{\alpha}$ in the quotient group $A^{*} / \mathbb{K}^{*}$ equals 3 as well. Similarly, one has $\xi^{p} \in \mathbb{K}$, and since $\xi \notin \mathbb{K}$, we conclude that for any $r<p$ one has $\xi^{r} \notin \mathbb{K}$. Thus the order of the image $\hat{\xi}$ of $\xi$ in $A^{*} / \mathbb{K}^{*}$ equals $p$. Furthermore, we have

$$
\hat{\xi} \hat{\alpha}=\hat{\alpha} \hat{\xi}^{d}
$$

These are exactly the defining relations of the group $G_{p}$. This means that the group $\hat{G}_{p}$ generated by $\hat{\xi}$ and $\hat{\alpha}$ in $A^{*} / \mathbb{K}^{*}$ is a quotient of $G_{p}$ such that $\hat{G}_{p}$ contains an element of order $p$ and an element of order 3 . Hence $\hat{G}_{p} \cong G_{p}$.

We see that the elements $\hat{\xi}$ and $\hat{\alpha}$ generate a subgroup isomorphic to $G_{p}$ in $A^{*} / \mathbb{K}^{*}$. It remains to recall that for a Severi-Brauer surface $S$ corresponding to $A$ one has $\operatorname{Aut}(S) \cong A^{*} / \mathbb{K}^{*}$, see for instance [ShV18, Lemma 4.1].

It would be interesting to obtain a complete classification of finite groups acting on non-trivial Severi-Brauer surfaces, similarly to what was done for conics in GA13. Also, we point out that for a given number field $\mathbb{K}$ there is only a finite number of finite groups $G$ such that there exists a Severi-Brauer surface over $\mathbb{K}$ with an action of $G$, cf. PSh14, Theorem 1.4]. This follows from the observation that any group $G$ like this acts faithfully on the anticanonical linear system of the Severi-Brauer surface, and thus is embedded into $\mathrm{PGL}_{10}(\mathbb{K})$. On the other hand, the latter group contains only a finite number of finite subgroups up to isomorphism by the (generalized) theorem of Minkowski, see for instance [Ser07, Theorem 5]. I do not know the answer to the following question.

Question 3. Does there exist an (infinite) extension $\mathbb{K}^{\prime}$ of $\mathbb{Q}$ and a non-trivial SeveriBrauer surface $S^{\prime}$ over $\mathbb{K}^{\prime}$ such that the group $\operatorname{Aut}(S)$ contains all the groups $G_{p}$ ?

Acknowledgements. I am grateful to S. Gorchinskiy and D. Osipov for useful discussions.

\section{REFERENCES}

[Cas67] Algebraic number theory. Edited by J. W. S. Cassels and A. Fröhlich. Academic Press, London; Thompson Book Co., Inc., Washington, D.C., 1967.

[GA13] M. Garcia-Armas. Finite group actions on curves of genus zero. J. Algebra, 394:173-181, 2013.

[GS06] Ph. Gille and T. Szamuely. Central simple algebras and Galois cohomology, volume 101 of Cambridge Studies in Advanced Mathematics. Cambridge University Press, Cambridge, 2006.

[GSh18] S. Gorchinskiy and C. Shramov. Unramified Brauer group and its applications, volume 246 of Translations of Mathematical Monographs. American Mathematical Society, Providence, RI, 2018.

[Pop14] V. Popov. Jordan groups and automorphism groups of algebraic varieties. Automorphisms in birational and affine geometry, 185-213, Springer Proc. Math. Stat., 79, Springer, Cham, 2014.

[PSh14] Yu. Prokhorov and C. Shramov. Jordan property for groups of birational selfmaps. Compositio Math., 150(12):2054-2072, 2014.

[Ser07] Jean-Pierre Serre. Bounds for the orders of the finite subgroups of $G(k)$. In Group representation theory, pages 405-450. EPFL Press, Lausanne, 2007. 
[Sh20] C. Shramov. Birational automorphisms of Severi-Brauer surfaces. Sb. Math., 211(3):466-480, 2020 .

[ShV18] C. Shramov and V. Vologodsky. Automorphisms of pointless surfaces. ArXiv e-print, 1807.06477, 2018.

[Ste89] L. Stern. On the norm groups of global fields. J. Number Theory, 32(2):203-219, 1989.

Steklov Mathematical Institute of Russian Academy of Sciences, 8 Gubkina st., Moscow, 119991, Russia

E-mail address: costya.shramov@gmail.com 